\title{
RETROSPECTIVE ANALYSIS OF 52 CASES OF ENTERIC FEVER IN A TERTIARY CARE HOSPITAL IN DHAKA CITY
}

\author{
MUTANABBI $\mathrm{M}^{1}$, ISLAM KA ${ }^{2}$, HELAL MA², MAMUN HA ${ }^{2}$, QUADER ${ }^{3}$, AHMED S ${ }^{4}$
}

\begin{abstract}
:
Enteric fever remains a serious problem in developing countries. School aged children and young adults are mainly the sufferer. Salmonella typhi infection results in a clinical syndrome that varies widely in presentation; especially in new antibiotic era. Records of 52 enteric fever cases who were admitted to BSMMU, from July 2007 to June 2008 was reviewed and information was recorded in a prescribed form. Descriptive analysis was done through SPSS. Mean age was $6.7 \pm 4$ years and male: female ratio was 1.2: 1. Most common presenting features were fever in 100\% cases, abdominal pain $40 \%$ cases, vomiting $35 \%$ and diarrhea, constipation $10 \%$ cases. Hepatomegaly was present in $71 \%$ and splenomegaly in 52\% cases. In our study32\% of children were under 5 years; and the result is similar with some other studies. 7,8 Typhoid fever is still a disease which is difficult to diagnose. In some cases of delayed response antibiotic may be required for longer duration where patients became afebrile after 10 days or more from starting antibiotic.
\end{abstract}

Key words: enteric fever, children, presentation

\section{Introduction:}

Enteric fever is an important public health problem in many of the developing countries. Estimates of the global burden of typhoid fever suggest an annual incidence of 12.5 million cases, with three-quarter occurring in Africa and South-East Asia. ${ }^{1,2}$ The incidence of typhoid fever is considered to be low in $1^{\text {st }}$ few years of life, peaking in school age children and young adults and then falling in the middle age..$^{2,3}$ Various organs have been affected in the course of enteric fever; and there is a wide array of presentations varying from nonspecific febrile illness to one of the severe life threatening illness. ${ }^{2,4}$ Emergence of MDR $\mathrm{S}$ typhi is a concern about response to treatment among treating physician. ${ }^{4}$

Salmonella typhi infection results in a clinical syndrome that varies widely in severity. At onset fever, malaise, flu like symptoms with dull frontal headache are common. The fever is initially low grade, and rises gradually and become high grade and sustained by second week. Often symptoms include poorly localized abdominal pain and dry cough. Physical signs are few - coated tongue, abdominal tenderness, rose spot, relative bradycardia and hepato-splenomegaly.

The epidemic nature of the disease in Bangladesh sufficiently warrants a review of the special features of the disease in children. If appropriate antibiotic is given fever gradually fall over 3 days. Due to emergence of MDR species and epidemiological aspect this study was conducted to see the presentation, outcome and response to antibiotic of enteric fever cases of BSMMU.

\section{Materials and methods:}

We reviewed all patients of Paediatric medicine unit 1 of BSMMU who were admitted over the period from July 2007 to June 2009. BSMMU is a referral hospital in Dhaka city where typhoid fever is endemic. ${ }^{5}$ In this ward children aged 1 month to 15 years were admitted. And cases were selected with strong clinical suspicion of enteric fever - like high continued fever for 7 days or more, along with one or more clinical features suggestive of enteric fever - like abdominal pain, vomiting, constipation, loose motion, abdominal distension, tenderness and hepato-splenomegaly. Other febrile illness was excluded. Patients were investigated to confirm the diagnosis and exclude other diagnosis and for any complications. Investigations were CBC, Widal test, blood culture $\&$ sensitivity, ALT, S bilirubin. Final diagnosis was considered with suggestive clinical features and positive Widal test or positive blood culture. All patients were treated with injectable antibiotics and

1. Assistant Professor, Department of Pediatrics, BSMMU

2. Consultant, Department of Pediatrics, BSMMU

3. Medical Officer, Department of Medicine, BSMMU

4. Assistant Professor, Department of Medicine, BSMMU

Bangladesh J Medicine 2010; 21 : 71-73 
followed up regularly. Antibiotic were continued up to 5 days after patient became afebrile. Patients were discharged after complete antibiotic course and followed up 2 week after discharge.

\section{Results:}

Records of A total 52 cases of typhoid fever were analyzed. Mean age was $6.7 \pm 4$ years. And male: female ratio was 1.2: 1 . Most common presenting features were fever $100 \%$ cases, abdominal pain $40 \%$ cases, vomiting $35 \%$ and constipation $10 \%$ cases. About physical findings $71 \%$ had hepatomegaly and $52 \%$ had splenomegaly.

Table-I

Symptoms and signs of patients at presentation

\begin{tabular}{lclc}
\hline $\begin{array}{l}\text { Symptoms } \\
\text { Features }\end{array}$ & $\begin{array}{c}\text { Signs } \\
\text { patients }\end{array}$ & Features & $\begin{array}{r}(\%) \text { of } \\
\text { patients }\end{array}$ \\
\hline Fever & 100 & Abdominal distention & 19 \\
Abdominal pain & 40 & Abdominal Tenderness & 39 \\
Vomiting & 35 & Hepatomegaly & 71 \\
Dirrhoea & 19 & Splenomegaly & 52 \\
Constipation & 10 & Rose spot & 2 \\
Cough & 8 & & \\
\hline
\end{tabular}

Table-II

Laboratory investigations

\begin{tabular}{lc}
\hline Findings & \% of patients \\
\hline Anemia & 36 \\
Widal Test positive & 69 \\
Blood culture positive & 17 \\
\hline
\end{tabular}

All patients received injection ceftriaxone and resolution of fever showed a wide range of $1-15$ days with mean 6.5 days. During this study period no death occurred due to typhoid fever.

\section{Discussion:}

Salmonella typhi infection remains a serious problem in developing countries and a major cause of mortality and morbidity. In our study $32 \%$ of children were under 5 years; and the result is similar with some other studies. ${ }^{7,} 8$ In our series dirrhoea was predominant gastrointestinal symptoms than constipation, and in accordance with other studies. ${ }^{8}, 9$ Typhoid is still difficult to diagnose, particularly in infant, Matheu et al reported 10 patient in their series presenting with mild illness like fever and cough. ${ }^{10}$ In our study $8 \%$ children presented with fever and cough.
In a study febrile convulsion was presenting symptom in $20 \%$ of the patients. ${ }^{2}$ In our study none of the patient presented with seizure. In our study hepatomegaly was higher than splenomegaly (71\% vs. $52 \%$ ), which is accordance with other studies. ${ }^{2}, 11$ Ohel and Latitan reported that, in their series hepatomegaly was almost twice as frequent as splenomegaly. 8,12 In another study in Turkey, Kanra et al found $17 \%$ encephalopathy, $73 \%$ hepatitis, $4 \%$ pneumonia, 4\% gastrointestinal bleeding; but we did not find similar features. Some of our children required antibiotics for longer duration even up to 23 days. In case of delayed responders, we did not change the antibiotics when the patients were seen to be stable i.e. not deteriorating clinically and also isolates were found to be sensitive to the given antibiotic. No fatal out come occurred in this series.

\section{Conclusion:}

Enteric fever is variable in presentation, none of the investigations is conclusive; sensitivity of widal test is slight higher but specificity is low and both sensitivity and specificity of blood culture are low. So Knowledge of variable presentations could help in diagnosis. It appears that in case of appropriate antibiotic we can wait safely for a longer period; here we waited up to 15 days for convalescence and no harm occurred. Rather frequent change of antibiotics may linger the illness and may lead to antibiotic resistance.

\section{References:}

1. Kiden Ya V, Ferson MJ. Typhoid and paratyphoid fever in south-east Sydney, 1992-1997. Commu Dis Intell. 2000; 24: 233-236.

2. Yaramis A, Yildirim I, Katar S, Ozbec MN, Yalcin I, Taj MA, Hosogu S. Clinical and Laboratory presentation of typhoid fever. Int Pediatr. 2001; 16(4): 227-231.

3. 'Salmonella' Infection: Clinical, immunological and molecular aspects, ed Pierto Mastruni and Duncan Muskell, Published by Cambridge university press, 2005.

4. Dimetro VT, Udo EE, Albaksam O, Al-Shehab S, Kilani A, Shehab M, Al- Nakkas A. Clinical and microbiological investigations of typhoid fever in a infectious disease hospital in Kawait. J Medical Microbiol. 2007; 56: 538-44.

5. Shaha SK, Baqui AH, Hanif M, Darmstadt GL, Amin MR, Nagatake T, Shantosam M, Blake RE. Typhoid fever in Bangladesh: Implications for vaccination policy. Pediatr Infec Dis J. 2001; 20(5): 521-24. 
6. Pang T, Bhutta ZA, Finlay BB, Altwegg M.Typhoid fever and other salmonelosis; a continuing challenge. Trends microbial. 1995; 3: 253-55.

7. Johnson AO, Aderele WI. Enteric fever in childhood. J Trop Med Hyg. 1981; 84: 29-35.

8. Oh HML, Masayu Z, Chew SK. Typhoid fever in hospitalized children in Singapore. J Infect.1997; 34: $237-42$.

9. Yap HF, Puthucheary SD. Typhoid fever in children - a retrospective study of 54 cases from Malaysia. Singapore Med J. 1998; 39: 260-62.
10. Methue J, Henning K, Bel E, Friede T. Typhoid fever in New York city 1980 through 1990. Arch Intern Med. 1994; 154: 1713-18.

11. Secmeer G, Karna G, Cemeroglu AP, Ozen H, Ceyhan M, Ecevit Z. Salmonellae typhi infection. A 10 year retrospective study. Turk J Pediatr. 1995; 37: 33941.

12. Laditan AA, Alaosa K. Problem in the clinical diagnosis of typhoid fever in children in topics. Ann trop Paediatra. 1981; 1: 191-15. 